\title{
Administration et dépendance dans un État de facto : les ambivalences de l'étatisation à Chypre du Nord
}

Administration and Dependence in a de facto State: the Ambivalence of the statization in Northern Cyprus

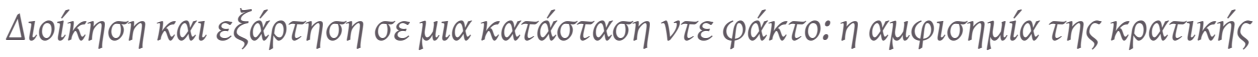

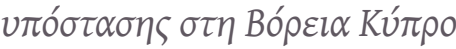

\section{Mathieu Petithomme}

\section{(2) OpenEdition}

\section{Journals}

Édition électronique

URL : https://journals.openedition.org/ceb/13597

DOI : 10.4000/ceb.13597

ISSN : 2261-4184

\section{Éditeur}

INALCO

Édition imprimée

ISBN : 9782858313341

ISSN : 0290-7402

\section{Référence électronique}

Mathieu Petithomme, «Administration et dépendance dans un État de facto : les ambivalences de

l'étatisation à Chypre du Nord », Cahiers balkaniques [En ligne], 46 | 2020, mis en ligne le 25 février 2020, consulté le 06 juillet 2021. URL : http://journals.openedition.org/ceb/13597 ; DOI : https:// doi.org/10.4000/ceb.13597

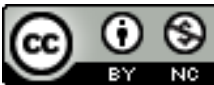

Cahiers balkaniques est mis à disposition selon les termes de la Licence Creative Commons Attribution - Pas d'Utilisation Commerciale 4.0 International. 


\title{
Administration et dépendance dans un État de facto : les ambivalences de l'étatisation à Chypre du Nord
}

\author{
Administration and Dependence in a de facto State: the \\ Ambivalence of the statization in Northern Cyprus \\ $\Delta \iota 0 i x \eta \sigma \eta x \alpha \iota \varepsilon \xi \alpha \dot{\alpha} \rho \eta \sigma \eta \sigma \varepsilon \mu l \alpha x \alpha \tau \dot{\alpha} \sigma \tau \alpha \sigma \eta \nu \tau \varepsilon \phi \alpha \dot{x} \tau 0: \eta$

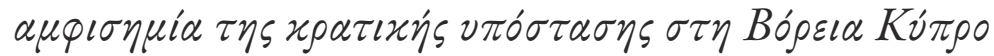

Mathieu Petithomme Université de Franche-Comté

Depuis son indépendance autoproclamée en 1983, la «République turque de Chypre du Nord» (RTCN, Kuzey Kibris Türk Cumburiyet, KKTC en turc), soutenue par la présence de 30000 militaires turcs, met en avant son autonomie vis-à-vis du Sud de l'île. À travers sa résolution 541 du 18 novembre 1983, le Conseil de sécurité de l'ONU, de même que la Grèce et la République de Chypre, considèrent que cette entité reconnue uniquement par la Turquie «n'a pas d'existence légale ». Mais sur le terrain, un gouvernement et des institutions parlementaires ont été institués, tandis que des partis fédéralistes et nationalistes s'opposent sur les politiques sociales et économiques à mener, sur la nécessité ou non de réunifier l'île et ses modalités potentielles. Une certaine quiétude au quotidien coexiste avec une militarisation de l'espace de ce territoire contesté. L'embargo économique imposé par la République de Chypre, puis par les grandes puissances, la CEE et l'UE après 1974, a plongé le territoire dans une crise profonde durant les années 1980 et poussé de nombreux Chypriotes turcs vers l'exil $^{1}$.

1. Bertrand, 2000, p. 59-81. 
Malgré l'absence de reconnaissance internationale, les élites nationalistes ont peu à peu créé une administration dont les dynamiques internes demeurent très méconnues. En contrepied des travaux géopolitiques sur le conflit chypriote et les processus de négociations, en se fondant sur une enquête de terrain ${ }^{2}$, cet article propose une «ethnographie de l'État» en s'intéressant au processus concret d'étatisation et à l'imaginaire du pouvoir, c'est-à-dire aux représentations que se font les Chypriotes turcs de leur propre administration et de leur «État de facto $^{3} \gg$. Mes entretiens avec des agents d'institutions administratives (ministère de l'Intérieur, de l'Économie, des Affaires étrangères et du Service des passeports) et du secteur public (enseignants et policiers notamment) sont mobilisés dans une démarche ethnographique où j'essaie de proposer un point de vue de l'intérieur de la genèse et du fonctionnement concret de l'administration chypriote turque, tout en restituant les comportements et les représentations des acteurs de l'État.

La RTCN ne doit pas être appréhendée comme une anomalie politique, une entité dont les pratiques administratives seraient pensées en opposition avec celles des autres États du système international. Les Chypriotes turcs se sont inspirés des modèles d'administration et des pratiques bureaucratiques des États légalement reconnus, notamment de la Grande-Bretagne, de la République de Chypre et de la Turquie. Sa structure étatique ne relève donc pas de la « tradition » ou d'une logique culturelle complètement distincte de celles de l'ancienne puissance coloniale et de ses voisins. Quelles représentations les Chypriotes turcs ont-ils de leur propre administration? Ma thèse est que l'étatisation d'un territoire non reconnu et dépendant du soutien politique et militaire d'un autre État engendre des représentations ambivalentes de ses «citoyens» à son égard, partagés entre révérence et dépréciation. L'isolement diplomatique et économique de la RTCN renforce une situation de dépendance des Chypriotes turcs vis-à-vis de leur administration qui engendre déférence et loyauté et peut déboucher sur des relations clientélistes. Mais la dépendance de leur État à l'égard de la

2. Cet article se fonde sur une enquête menée durant quatre mois en février 2012, mars/avril 2013, mars/avril et novembre 2014 à Lefkoşa (Lefkosia) et Gazimağusa (Ammochostos) qui permit de recueillir des observations ethnographiques, et plus de 80 entretiens (en anglais ou en turc avec des intermédiaires) avec des représentants politiques et des citoyens ordinaires.

3. Scott Pegg définit l' «État de facto » comme «un leadership politique organisé qui a acquis une capacité suffisante pour fournir des services gouvernementaux à une population au sein d'une aire territoriale donnée, sur laquelle un contrôle effectif est maintenu durant une période de temps significative», mais qui est dépourvu de reconnaissance internationale (PEGG, 1998, p. 26). 
Turquie et l'absence de changement dans le statut du territoire engendrent aussi une insatisfaction chronique et une conscience chez les citoyens des limites de la «souveraineté » de leur entité politique. Le rapport des Chypriotes turcs avec leur administration non reconnue peut donc être appréhendé à partir d'une anthropologie politique du don, puisque leurs attitudes s'insèrent dans le contexte d'une double relation de dépendance: ils sont dépendants vis-à-vis de leur administration, elle-même dépendante à l'égard de la Turquie.

Ils cherchent en effet par tous les moyens à exploiter leurs réseaux sociaux (torpil) afin d'entrer dans l' «administration d'État » (devlet dairesi) qui symbolise pour eux la promesse d'une stabilité salariale et sociale. Les mères de famille cherchent des beaux-fils fonctionnaires et les parents utilisent leurs «connexions» pour trouver un emploi à leurs enfants dans la fonction publique. Dans une économie sous embargo où les emplois dans le secteur privé demeurent limités et soumis à une pérennité incertaine, les «hommes de gouvernement (bükümet adamlar1) bénéficient d'un prestige social important. Pour autant, même si les Chypriotes turcs désirent intégrer la bureaucratie et si les fonctionnaires développent des attitudes de révérence et de loyauté à l'égard de leur administration, ils critiquent aussi l'oisiveté de leurs agents publics et développent une vision critique de la RTCN. Nombreux sont ceux qui considèrent leur État comme inefficace et trop dépendant de la Turquie pour être réellement fonctionnel. Néanmoins, même si les documents et les procédures administratives de la RTCN ne sont pas reconnus internationalement, ils créent du sens au quotidien pour les Chypriotes turcs qui demeurent très attachés à un secteur public et à un État qu' ils critiquent pourtant allégrement.

J'insère d'abord la problématique de l'étatisation d'un territoire non reconnu dans le cadre des travaux classiques d'anthropologie politique sur le don. Je retrace ensuite le processus d'étatisation de la RTCN, en montrant les origines historiques de la dépendance des Chypriotes turcs à l'égard de leur administration, puis de celle de la RTCN elle-même à l'égard de la Turquie. J'étudie enfin les affects créés par ces logiques de dépendance sur la légitimation sociale de l'étatisation, à travers la prévalence du clientélisme, d'une politisation des institutions et d'attitudes de résignation et de soumission à l'autorité.

\section{Étatisation, don et dépendance : une approche ethnographique}

Ethnographier un État non reconnu implique de s'intéresser notamment aux rapports qu'entretiennent les agents publics et à l'idée que se font les habitants de leur propre administration. La méthode ethnographique étudie les relations d'interdépendance entre des acteurs sociaux, soit parce qu'elles sont directement 
28 Politique et société à Chypre aujourd'hui

données à voir à l'ethnographe « au cours d'interactions de face à face auxquelles il participe et de cérémonies publiques auxquelles il assiste », soit parce qu'elles passent « par la médiation de dispositifs matériels et d'institutions dont il peut directement observer le fonctionnement et la genèse ${ }^{4}$. » L'analyse des interactions des fonctionnaires chypriotes turcs avec leur administration permet de mettre en avant le contexte de la «scène sociale » d'un État de facto, dans lequel elles prennent leur signification pour leurs acteurs. L'ethnographie me permet de montrer l'existence d'une double relation de dépendance des Chypriotes turcs, dont il s'agit d'expliquer les origines historiques, les modalités de persistance et les implications.

L'ambivalence des rapports des Chypriotes turcs avec leur administration peut être saisie grâce aux travaux classiques d'anthropologie politique sur le don, qui ont montré l'ambivalence de cet échange social. Il peut impliquer une alliance comme dans la kula observée en Mélanésie par Bronislaw Malinowski (1922). Il peut aussi médiatiser une rivalité politique, comme l'illustre l'étude de Franz Boas de la cérémonie du potlatch chez les Indiens Kwakiutl de l'Est de l'Alaska, qui met en scène des luttes de générosité, des «prestations totales de type agonistique », dont le but est d'établir une hiérarchie entre les représentants de différents groupes, puisque le plus fort sera celui qui offrira le plus de richesses aux autres, y compris en les détruisant ${ }^{5}$. Selon Annette Weiner, en manifestant leur mépris de la richesse, c'est l'honneur des protagonistes, leur mana, qui est engagé dans ces luttes qui permettent de définir le sens des relations de dépendance et le pouvoir symbolique et concret de certains acteurs plus généreux sur les autres. Weiner parle du «paradoxe de donner tout en retenant», dans le sens où le don, s'il est reconnu comme tel par le donataire, engendre une nécessité de rendre et une relation de dépendance ${ }^{6}$. Pour Marcel Mauss, le don engendre aussi une dette personnelle qui asservit, puisque des échanges de faveur «en théorie volontaires» sont «en réalité obligatoirement faits et rendus ${ }^{7}$ ». Le don instituerait $\ll$ la triple obligation de donner, de recevoir et de rendre ${ }^{8} »$. Pour Pierre Bourdieu, le laps de temps entre le don et le contre-don, «permet au donateur de faire violence au donataire - contraint de rester le débiteur du donateur - et de masquer cette violence sous une apparence de générosité sans

4. WeBER, 2007, p. 29.

5. BOAS, 1897 , p. 362-363.

6. WEINER, 1992.

7. Mauss, 1924-1925, p. 65 et 139.

8. Ibid. p. 87. 
calcul'. » C'est ce qui rapprocherait le don de la dette, puisque celui qui reçoit deviendrait souvent l'obligé du donateur. Il faudrait définir «la séquence d'interactions », entre «le premier don » et le «contre-don», pour saisir s'il s'agit d'une alliance équilibrée, d'une dépendance ou d'une rivalité entre deux protagonistes ${ }^{10}$.

Ces travaux classiques permettent de comprendre qu'en acceptant une dépendance à l'égard d'une administration soutenue par un autre État, les Chypriotes turcs sont engagés malgré eux dans une relation asymétrique. Comme l'écrit l'ethnologue Maurice Godelier, «le don crée des dettes qui ne s'effacent pas $^{11} \gg$. En fondant un État autoproclamé dont l'administration n'existe que grâce au soutien politique et militaire de la Turquie, les Chypriotes turcs ont reçu «un don sur le dos » qui n'est à aucun degré désintéressé: en échange du soutien de leur administration (elle-même soutenue par la Turquie) qui leur fournit des emplois et une stabilité salariale dans un contexte d'embargo économique et d'isolement international, ils deviennent redevables à l'égard de la Turquie, dans l'obligation de manifester «une reconnaissance de la dette de vie », qui perdure par «la commémoration toujours vivante de l'alliance que contracte le don ${ }^{12}$ ». Dans l'impossibilité « de rendre » l'appui militaire et l'aide financière que la Turquie leur « donne », ils sont contraints à une « subordination symbolique » et à une allégeance au nationalisme turc. Les économistes Çetin Uğural et Ferdi Sabit Soyer évoquent à ce propos une «économie de la gratitude » (Şükran Ekonomisi) où un discours politique qui exagère le rôle de l'«aide turque» domine l'espace public afin de donner le sentiment à la population que le maintien du soutien de la Turquie est nécessaire ${ }^{13}$.

Pour les agents publics de l'État, dont les positions dominantes donnent accès à un salaire et à des privilèges synonymes de distinction sociale, la dépendance à l'égard de leur administration s'exprime par la loyauté, le conformisme et la soumission à l'autorité. Les Chypriotes turcs utilisent d'ailleurs l'expression «prendre l'État à bras le corps» (devlet belden bağlanurlar) pour critiquer des fonctionnaires dépendants de l'État par le « ventre ${ }^{14} »$. En resituant les relations de dépendance qui lient les agents publics à leur administration, et

9. Bourdieu, 1991, p. 27.

10. Weber, 2007, p. 29.

11. GODELIER, 1996, p. 15.

12. Rosbapé, 1993, p. 15-33 ; Caillé \& Godbout, 1992, p. 21.

13. UĞURAL \& SOYER, 1998.

14. Navaro-Yashin, 2006, p. 282. 
les Chypriotes turcs à leur État, dans le contexte d'un partenariat historique avec la Turquie, j'invite à reconsidérer l'État comme un «État affectif» pour reprendre l'expression d'Ann Laura Stoler, dans le sens où les bureaucraties, loin de constituer uniquement des appareils institutionnels dominés par la rationalité, contribuent aussi à produire des émotions chez leurs agents, comme l'a bien montré l'étude ethnographique magistrale de Vincent Dubois ${ }^{15}$. À Chypre du Nord, dans le contexte d'un État de facto au statut juridique contesté, le désir des Chypriotes turcs d'intégrer la bureaucratie va de pair avec une critique de son ineffectivité et de sa dépendance à l'égard d'un État tiers. Comme le rappellent Jacques Lagroye et Johanna Siméant, «la part affective de l'attachement à des institutions et aux pratiques qui s'y déploient apparaît comme une dimension centrale de leur légitimation ${ }^{16}{ } \gg$. C'est cet aspect subtil et souvent négligé des «émotions morales évoquées par Didier Fassin, que je propose d'étudier à travers les rapports ambivalents des Chypriotes turcs à leur État, partagés entre désir et allégeance, ressentiment et frustration ${ }^{17}$.

\section{Étatisation et dépendance historique des Chypriotes turcs à l'égard de leur administration}

La RTCN a été instituée par un processus d'étatisation en plusieurs étapes. Pour les nationalistes, la possibilité de partager un gouvernement commun avec les Chypriotes grecs a disparu depuis 1963, après l'expulsion des dirigeants chypriotes turcs des institutions, la marginalisation sociale puis le repli de leur communauté dans 42 enclaves. Alors que les Chypriotes grecs perçoivent l'«invasion » turque du 20 août 1974 comme le processus déclencheur de la partition et de leur «tragédie nationale», le calendrier mémoriel chypriote turc mobilise la révélation du plan Akritas, le projet des 13 amendements constitutionnels de Makarios et les heurts du «Noël Sanglant» du 21 décembre 1963, pour justifier la partition de l'île et la création d'une administration d'État autonome ${ }^{18}$. Les Chypriotes turcs ont été représentés par l'«Association de la minorité turque de Chypre» sous la colonisation britannique à partir de 1943, puis par l'《organisation turque de résistance » (Türk Mukavemet Teşkilatı, TMT) après 1957, la « Fédération des associations

15. Stoler, 2004, p. 4-20, Dubois, 1999.

16. Lagroye \& SimÉant, 2003, p. 54-56.

17. FASSIN, 2013, p. 249-267.

18. Papadakis, 1993, p. 130-148. 
turques de Chypre » et les municipalités séparées dans les grandes villes de l'île en $1958^{19}$. Ils furent dotés d'institutions propres et de pouvoirs importants dans la République bicommunautaire établie par la Constitution de 1960. En 1963, le «Comité général » chypriote turc fut remplacé par l' « Administration chypriote turque provisoire ( 1964) puis par l'«État turc fédéré de Chypre » en $1975^{20}$. La dépendance des Chypriotes turcs à l'égard de leur administration trouve ainsi son fondement historique dans leur situation d'isolement et de précarité sous la double menace physique des activistes de l'«organisation nationale des combattants chypriotes » (Ethniki Organosis Kyprion Agoniston, EOKA), et des combattants de la TMT entre 1963 et 1974. Ils furent alors peu à peu licenciés par leurs employeurs, quittèrent leur travail de leur propre initiative ou trouvèrent simplement trop dangereux de se rendre dans les zones chypriotes grecques ${ }^{21}$. Tuncay Halil, ancien membre de la TMT dans les années 1960, évoque bien le processus de ségrégation puis de formation d'une administration autonome:

Après décembre 1963, lorsque notre vice-président Fazil Küçük et nos ministres furent marginalisés des réunions du cabinet, un choix politique fut pris de boycotter les institutions pour marquer notre désaccord. Les députés et les fonctionnaires d'État chypriotes turcs encore en poste se retirèrent d'eux-mêmes et rejoignirent ceux qui avaient été empêchés d'accéder à leurs bureaux. Küçük forma un «Comité général » réunissant l'ensemble des élites pour protéger notre communauté et administrer les enclaves ${ }^{22}$.

Deniz Vardar, un autre ancien combattant de la TMT, juge aussi sévèrement l'attitude des dirigeants chypriotes grecs:

Au lieu de renouer le dialogue, ils s'approprièrent la police, le gouvernement, l'administration, la justice et la Chambre des représentants. Ils confisquèrent le pouvoir. L'ensemble des institutions « bicommunautaires $»$ devinrent chypriotes grecques ${ }^{23}$.

19. Copeaux \& Mauss-Copeaux, 2005, p. 27.

20. Navaro-Yashin, 2012, p. 39.

21. BRYANT, 2004, p. 43.

22. Autant que possible, je précise la fonction, l'âge des enquêtés, le lieu et la date de l'entretien. Ici, 72 ans, avenue Ikinsi Selim, Lefkoşa, 6/2/2012.

23. 62 ans, secrétaire général d'une association des anciens combattants de la TMT, avenue Ikinsi Selim, Lefkoşa, 6/2/2012. 
32 Politique et société à Chypre aujourd'hui

Ceux qui continuèrent à travailler avec les Chypriotes grecs furent présentés comme des «traîtres » et des «antipatriotes » (vatan düşmanı) et menacés de représailles par les activistes de la TMT, de telle sorte que lors de la proclamation de l'«Administration chypriote turque provisoire » en avril 1964, plus aucun Chypriote turc ne travaillait dans les institutions républicaines ${ }^{24}$. Poussés de force par les miliciens de la TMT à quitter les quartiers, les villages mixtes et les petits villages à majorité turque difficiles à protéger, les Chypriotes turcs furent regroupés dans des enclaves où ils devinrent les sujets d'une administration parallèle. Les restrictions à la liberté de circulation au-delà des enclaves, la peur des attentats et des attaques de l'EOKA en se rendant en zone chypriote grecque, ou celle de subir les intimidations et les représailles des miliciens de la TMT à leur retour, les conduisirent peu à peu, comme leurs dirigeants avant eux, à quitter leurs emplois antérieurs. Au déracinement social lié au déplacement géographique forcé, s'ajoutèrent une ségrégation spatiale et une paupérisation économique, qui engendrèrent une situation concrète de dépendance des déplacés à l'égard des nouvelles structures administratives créées dans les enclaves et dirigées par les membres de la TMT. Yılmaz, un retraité de Famagouste, évoque l'émergence de ce lien de dépendance dans la formation des nouvelles institutions :

Les combattants de la TMT proposaient des postes administratifs dans les enclaves pour inciter les notables et les classes moyennes à s'y installer. Ils faisaient des promesses d'emplois et de salaires. Ils utilisaient la peur ou la menace contre ceux qui voulaient rester dans leurs villages. Ils incitaient les gens à se regrouper et à rejoindre la résistance. Ceux qui ont obtenu des postes sont devenus redevables envers l'organisation. Ils se sont socialisés dans un environnement très nationaliste lié au conflit, aux menaces et au climat de peur qui régnait ${ }^{25}$.

Les structures paraétatiques qui émergèrent durant cette période furent d'abord une extension de la structure organisationnelle de la TMT avec ses hiérarchies, son nationalisme et sa culture de la discipline et du secret ${ }^{42}$. Selon Richard Patrick, «le leadership de facto au niveau du village et du quartier, du district et de la communauté dans son ensemble, fut assumé par les combattants. La structure de gouvernement de la communauté était une synthèse militaro-

24. Loizos, 2008, p. 32.

25.71 ans, rue Balaban, Gazimağusa, 9/4/2014. 
civile $^{26} \gg$. L' «État-TMT » qui émergea fut donc fondé sur une hiérarchie stricte des rôles, la toute-puissance d'organes de contrôle et de surveillance et l'imposition d'une loyauté et d'un nationalisme sans faille demandée à ses agents. L'engagement dans la TMT donnait à ses membres un prestige social, l'accès à un emploi et à de nombreux avantages matériels comme la priorité dans la distribution des biens pillés aux Chypriotes grecs (maisons, terres, meubles, etc.), ou le contrôle de l'aide humanitaire distribuée par l'UNFICYP. L'accès privilégié à ces bénéfices sociaux, politiques et économiques et la militarisation des enclaves permirent d'instituer une administration autoritaire d'essence «néo-patrimoniale» au sens de Jean-François Médard, à savoir qui permet à ses agents de cumuler des positions de pouvoir avec des leviers d'enrichissement personnel ${ }^{27}$. Mais en échange d'un emploi dans les forces de sécurité, d'un poste administratif ou d'un salaire, les règles de fonctionnement de la nouvelle bureaucratie d'État et les pressions de ses agents les plus influents, impliquèrent en retour une obéissance et une loyauté au projet de construction nationale. Le développement des tensions intercommunautaires facilita l'émergence d'un régime qui institua une atmosphère de surveillance, de contrôle et de discipline, en incitant les citoyens, par la propagande, les intimidations et les menaces, à partager l'idéal nationaliste, «la partition ou la mort» (Ya Taksim Ya Ölüm). De nombreux Chypriotes turcs, particulièrement ceux soupçonnés d'être des « communistes » et leurs familles, firent face à la répression des nationalistes, une situation particulièrement pesante dont se remémore l'opposant Alpay Durduran :

Les lois et la justice républicaines des institutions de 1960 ne furent plus appliquées dans les enclaves. Nous étions à la merci des combattants de la TMT qui prétendaient nous défendre, mais qui exerçaient surtout une répression en toute impunité contre leurs opposants ou ceux qu'ils soupçonnaient de complaisance avec l'ennemi. Des gens ont été assassinés pour rien, parfois pour des conflits de voisinage ou un mot de trop ${ }^{28}$.

Malgré le caractère autoritaire de cette administration, le contexte d'enclavement, d'embargo économique et de pénurie, explique la dépendance historique des Chypriotes turcs vis-à-vis d'une bureaucratie perçue comme jouant la fonction d'un employeur et d'un système de protection sociale à grande

26. PATrick, 1976, p. 84.

27. MÉdARD, 1992, p. 3-24.

28. 75 ans, secrétaire pour les affaires internationales, parti de la Nouvelle Chypre (Yeni Kibris Partisi, YKP), rue Tanzimat, Lefkoşa, 14/2/2012. 
échelle. Ses recrutements pléthoriques s'expliquent par la volonté politique d'élargir la base nationaliste, de s'assurer de la loyauté de ses agents et de limiter les contestations potentielles, mais aussi par une nécessité sociale de compenser la rareté des emplois marchands. Le fait que les Chypriotes turcs vivent toujours aujourd'hui dans un contexte d'isolement international et d'embargo économique plus de cinquante ans après la création des enclaves explique leur attachement pragmatique et historiquement ancré à leurs postes au sein des administrations de la RTCN. Selon Mustafa Tolga, secrétaire général de l'association des retraités du secteur public (Kibris Türk Emekliler Cemiyeti), l'ensemble des postes au sein des ministères, des mairies, des écoles, de la police et des différents services administratifs représentent environ $20 \%$ des emplois de la RTCN, et font vivre directement ou indirectement plus du tiers de la population ${ }^{29}$. Selon la KTAMS, l'union des employés du secteur public, la RTCN comptait environ 13500 fonctionnaires en 2013 ${ }^{30}$. Şener Levent, éditorialiste du quotidien indépendant Afrika Gazetesi, explique l'attrait et la dépendance des Chypriotes turcs à l'égard de leur administration comme une réponse pragmatique à l'absence de perspectives:

Les Chypriotes turcs éduquent leurs enfants en leur vantant les mérites de devenir fonctionnaires, en leur disant que c'est la meilleure manière d'accéder à un emploi et de fonder une famille en restant ici, sans être obligé de s'exiler. Ils leur inculquent ces idées très jeunes et leur disent que pour cela, il faut faire profil bas, se soumettre à l'autorité et faire preuve de loyauté envers les chefs et l'organisation ${ }^{31}$.

Ce désir largement partagé d'entrer dans le secteur public demeure paradoxal, puisque c'est la conscience du caractère peu dynamique et viable du marché du travail local qui crée une survalorisation de la bureaucratie d'État. Cela engendre donc très souvent des attitudes conformistes et conservatrices des agents de l'État par rapport aux réformes potentielles et aux dysfonctionnements bureaucratiques. Lors de mes entretiens avec des employés de différentes administrations, j'ai noté une certaine intériorisation de la soumission propre aux rapports de domination, particulièrement répandue chez les «assistantes ». Leman, secrétaire administrative du ministère de l'Économie et de l'Énergie

29. 66 ans, rue Sarayonü, Lefkoşa, 13/2/2012.

30. «KTAMS' defends North Cyprus civil servants », Cyprus Mail, 15/10/2013, p. 16.

31. Avenue Osmanpaşa, Lefkoşa, 12/3/2014. 
(KKTC Ekonomi ve Ererji Bakanliği), me confia ainsi lors d'un entretien après sa journée de travail, dans un petit café à la sortie du ministère :

Je ne donne jamais mon point de vue à mes supérieurs. Je fais ce qu'ils me demandent sans discuter, même s'il s'agit de faire le plein d'essence de leur voiture ou d'aller chercher leurs enfants à l'école. En échange, je gagne mon salaire et on me laisse tranquille ${ }^{32}$.

Sa collègue Ece, qui travaille au sein du même ministère dans un autre service renchérit lors de notre conversation collective : «Il faut être dévouée. Ne jamais discuter les ordres. Si l'on fait cela, il n'y a pas de problèmes. Notre travail n'évolue pas trop, mais il est assuré3 ${ }^{3}$. »

La sociohistoire de l'administration chypriote turque permet ainsi de montrer que, deux décennies avant la création de la RTCN en 1983, le processus d'étatisation enclenché dans les enclaves chypriotes turques (1963-1974) établissait déjà les fondations de l'administration nord-chypriote, dont les traits principaux sont aujourd'hui encore observables: la dépendance à l'égard de l'administration, son surdéveloppement, son rôle de protection sociale dans un contexte de pénurie, et son encadrement par un complexe militaro-civil qui implique la loyauté de ses agents et des attitudes de soumission. Mais ce lien pragmatique et affectif particulier s'insère dans le contexte de la dépendance simultanée de la RTCN à l'égard de la Turquie, tant sur les plans militaire, politique et économique. Les salaires des fonctionnaires sont en effet dépendants de l'aide financière annuelle octroyée par le gouvernement turc à la RTCN. Si l'on ajoute les 30000 militaires directement payés par l'État turc aux membres de l'administration et leurs familles, une part très importante de la population «officieuse » de la RTCN (mais pas de la population «officielle») dépend donc financièrement de la Turquie.

\section{La dépendance de la RTCN à l'égard de la Turquie}

Les Chypriotes turcs entretiennent aussi des rapports ambivalents vis-à-vis de la Turquie, puissance «bienfaitrice » qui les a «sauvés de l'ennemi » et qui les «aide de façon désintéressée » selon Tahsin Ertuğruloğlü (DGP), autant d’actes de bravoure qui masquent pourtant l'exercice d'un pouvoir de domination ${ }^{34}$. La

32. Ministère de l'Économie et de l'Énergie, rue Idris Doğan, Lefkoşa, 21/3/2014.

33. Ibid.

34. 53 ans, président du parti de la Démocratie et de la confiance (Demokrasi ve Güven Partisi, DGP), avenue Osman Paşa, Lefkoşa, 8/2/2012. 
36 Politique et société à Chypre aujourd'hui

dépendance de l'administration chypriote turque à l'égard de la Turquie trouve ses origines dans l'expulsion des dirigeants chypriotes turcs des institutions de la République en 1963. Dès juillet 1958, les Chypriotes turcs purent administrer de façon autonome la «Municipalité turque de Nicosie », puis les municipalités séparées de Famagouste, Limassol, Paphos et Larnaca suite à la loi de 1959 sur les Comités municipaux turcs. L'article 173 de la Constitution de 1960 reconnut la légalité de ces cinq municipalités séparées, tout en octroyant aux Chypriotes turcs un quota de $30 \%$ des postes au sein de la fonction publique, même si leur communauté ne représentait que $18 \%$ de la population. Mais ces dispositions constitutionnelles furent unilatéralement remises en cause par les Chypriotes grecs. L'embargo économique imposé aux enclaves en janvier 1964 par le gouvernement chypriote grec coupa les circuits commerciaux et les échanges entre les deux communautés et précipita l'appauvrissement des Chypriotes turcs. C'est dans ce contexte que les 42 enclaves qui couvraient 1,6 \% du territoire de Chypre devinrent la base de la future administration chypriote turque ${ }^{35}$.

Ceux qui sont aujourd'hui âgés de plus de 60 ans, comme Hüseyin, un ancien artisan à la retraite de Famagouste, évoquent avec rancœur cette période associée aux privations, à la misère et à la peur :

La vie dans les enclaves était très dure. On ne pouvait pas circuler librement. Les combattants de la TMT nous obligeaient à rejoindre la résistance. Ils nous protégeaient, mais nous faisaient peur aussi. Ils contrôlaient tout, distribuaient les rations de nourriture et les aides en provenance du gouvernement turc. Il ne fallait surtout pas leur désobéir ${ }^{36}$.

Richard Patrick considère que l'aide financière de la Turquie devint justement plus directe et substantielle durant cette période pour compenser la paupérisation :

En 1968, le gouvernement turc injectait 8 millions de pounds [dix millions d'euros] par an dans l'économie chypriote turque. Cette manne servait à financer les programmes et les aides sociales, de même que les salaires des dirigeants chypriotes turcs, des fonctionnaires et de l'ensemble des combattants de la $\mathrm{TMT}^{37}$.

35. Attalides, 1979, p. 90.

36. $28 / 4 / 2014$.

37. PATRICK, 1976, p. 110. 
Le contexte de ségrégation géographique et de conflit intercommunautaire « rendit donc une population entière largement dépendante d'une administration d'État en formation soutenue par un autre État ${ }^{38}$ ».

L'ambivalence des représentations que se font les Chypriotes turcs de leur propre administration s'explique ainsi par leur dépendance historique concrète à l'égard de ses ressources, parallèlement à une conscience du caractère quelque peu artificiel de leur «État» non reconnu, construit sur une base territoriale contestée et grâce à l'appui militaire d'un autre État. À l'attachement et au désir d'intégrer la bureaucratie se mêle une distance critique à l'égard d'un «État virtuel d dépendant, et qui n'exerce donc qu'imparfaitement son rôle. Les fonctionnaires sont parfaitement conscients que leurs salaires dépendent de l'aide financière accordée par le gouvernement turc. Murat Kanatli, dirigeant du parti de la nouvelle Chypre (Yeni Kıbrıs Partisi, YKP), considère ainsi que « nos gouvernements successifs se sont habitués à mendier à Ankara. ${ }^{39}$ » Les employés du secteur public sont donc à la fois reconnaissants à l'égard de la Turquie, mais aussi critiques de l'incapacité de leurs gouvernants à s'émanciper du soutien turc et à créer un État viable économiquement et autonome.

Lors de mes entretiens avec des professeurs du lycée professionnel Sedat Semavi de Lefkoşa (Sedat Simavi endüstri Meslek Lisesi), j'ai entendu des discours quasi unanimes de gratitude à l'égard de la Turquie. Safiye Koyumcu, une professeure de géographie, pense ainsi que «la Turquie nous aide beaucoup. Sans elle, ce pays n'existerait pas $^{40} \gg$. Pour Ayse Hüsam, professeure de mathématiques:

Ce serait bien si notre gouvernement était plus autonome et avait plus de ressources. Ce sera peut-être possible avec la découverte du gaz. Mais pour l'instant, heureusement que nous pouvons nous appuyer sur le soutien de la Turquie ${ }^{41}$.

Reprenant inconsciemment des termes de la propagande officielle, Serhan Çağman, professeure d'éducation physique, déclara que «la Turquie est notre "mère-patrie" [anavatan], elle nous protège et nous aide à nous développer ${ }^{42} . \gg$ Depuis 1974, les nationalistes ne cessent en effet de présenter

38. Navaro-Yashin, 2012, p. 89.

39. 44 ans, rue Tanzimat, Lefkoşa, 14/2/2012.

40.33 ans, Lefkoşa, 3/4/2014.

41. 34 ans, Lefkoşa, 4/4/2014.

42.50 ans, Lefkoşa, 4/4/2014. 
38 Politique et société à Chypre aujourd'hui

l'armée turque comme celle qui aurait «sauvé» les Chypriotes turcs de l'extermination, et la Turquie comme une «bienfaitrice» qui les aiderait par solidarité envers un peuple-frère. Pour Yael Navaro-Yashin, cet «affect du remerciement» a d'ailleurs «été encouragé comme une politique par les administrations successives ${ }^{43}$.

Mais pour certains critiques comme le journaliste Şener Levent, «cette politique de la reconnaissance forcée» ne servirait qu'à «entretenir une illusion, car la Turquie tire plus de bénéfices économiques de ses liens avec la RTCN qu'elle ne l'aide financièrement ${ }^{44} \gg$. Même un nationaliste comme Metin Çatan (Demokrat Parti, DP), déplore la dépendance de l'État chypriote turc à l'égard de la Turquie, qu'il explique toutefois comme une nécessité dans le contexte d'isolement:

Le gouvernement ici est trop dépendant des décisions qui se prennent à Ankara. Le problème c'est que l'embargo a asphyxié notre indépendance économique. Comme la Turquie nous aide financièrement, il est difficile d'agir politiquement sans son accord ${ }^{45}$.

Les nationalistes reconnaissent parfois cette dépendance, mais la justifient comme un mal nécessaire en l'absence d'autre choix possible puisqu'ils refusent la réunification, perçue comme une absorption de leur entité par la République de Chypre, et que la communauté internationale ne reconnaît pas la RTCN. Pour Omer Adal, membre du bureau du DP :

Une Chypre unifiée signifierait un retour en arrière. Nous ne voulons pas être à nouveau traités comme des citoyens de seconde zone. Mieux vaut rester autonomes même s'il faut maintenir des liens très forts avec la Turquie ${ }^{46}$.

Alors que, pour les nationalistes, le maintien de l'autonomie de la RTCN est pensé dans son rapport avec la République de Chypre, les fédéralistes comme Murat Kanatli dénoncent une «autonomie fictive», puisqu'elle impliquerait «une intégration forcée avec la Turquie ${ }^{47} »$. Pour Izzet Izcandir,

43. Navaro-Yashin, 2012, p. 89.

44. "Partnership with Turkey could be more advantageous for TRNC", Cyprus Mail, $1 / 6 / 2009$, p. 2.

45. 64 ans, membre du bureau du DP, rue Server Somuncuoğlü, Lefkoşa, 6/2/2012.

46. 49 ans, rue Server Somuncuoğlü, Lefkoşa, 6/2/2012.

47. 44 ans, rue Tanzimat, Lefkoşa, 14/2/2012. 
secrétaire général du parti de Chypre unifié (Birleşik Kıbrıs Partisi, BKP), l'accent mis par les nationalistes sur l'«indépendance » de la RTCN répondrait à une stratégie de propagande visant à justifier la partition et à passer sous silence son manque de viabilité économique sans le soutien de la Turquie:

La RTCN n'est pas indépendante et tout le monde le sait. Les nationalistes évoquent sans cesse «l'État turc» de Chypre, «l'indépendance de la RTCN » et sa «reconnaissance internationale » prochaine. Ils parlent aussi de la Turquie comme de «notre État». La réalité, c'est que la RTCN est une création artificielle de la Turquie. Les Chypriotes turcs ne peuvent pas vivre isolés sans liens avec les Chypriotes grecs et avec l'extérieur ${ }^{48}$.

Les Chypriotes turcs, pleinement conscients, ont intériorisé dans leurs pratiques sociales l'existence d'une facette artificielle de leur État et de ses liens particuliers avec la Turquie. À l'école, les références sont ambivalentes: on apprend tant que les Turcs d'Anatolie et de Méditerranée ont vécu au sein d'une multitude d'États et ont coexisté au sein de l'Empire ottoman, présenté comme un Empire multinational, mais aussi qu'ils doivent s'identifier à l'héritage d'Atatürk et de la République de Turquie fondée en 1915, tout en présentant la RTCN comme l'État indépendant des Turcs de Chypre ${ }^{49}$. Avec le temps, les jeunes Chypriotes turcs apprennent à s'accommoder de ces allégeances nationales multiples et ambigües. Leurs relations assez conflictuelles avec les «Turcs» immigrés les ont amenés à prendre plus conscience de leurs particularismes, à se sentir plus «Chypriotes turcs», même si la propagande nationaliste récuse l'existence d'une telle identité et ne cesse de glorifier les liens avec la «mère-patrie ». L'utilisation politique des programmes éducatifs, de la télévision publique et des célébrations d'État par les nationalistes depuis 1974 ont toutefois largement permis de diffuser l'affect de reconnaissance envers la Turquie au sein de la population.

Nationalisme et politisation de la bureaucratie

À Chypre du Nord, c'est d'abord à travers l'extension du groupe familial et le réseau d'amitiés que l'individu entre en contact avec l'environnement économique et administratif dans lequel il négocie les contraintes et essaie de s'insérer socialement. La forte hiérarchie et l'organisation pyramidale de l'administration permet de répartir les rôles entre des instances distinctes, tout en facilitant l'exercice de la surveillance et du regard disciplinaire des chefs de

48. Rue Ibrahim Ramadan, Lefkoşa, 20/2/2012.

49. KIZILyÜREK, 2002, p. 432-435. 
service. La politisation de la bureaucratie se traduit par l'allégeance voulue ou contrainte des fonctionnaires à l' «idéologie d'État» qui justifie historiquement l'existence de la RTCN, et qui se fonde sur le présupposé que la partition et la création d'une entité politique autonome auraient résolu le conflit de 1974. Les fonctionnaires bénéficient de nombreux avantages sociaux, matériels et financiers qui créent une dépendance et renforcent leur attachement à l'administration et à l'État. Par ailleurs, la formation du corps administratif s'est accompagnée de tendances clientélistes et d'une forte politisation des institutions, puisque l'accès aux différents corps d'État, à la police, aux administrations publiques et aux postes d'enseignants s'effectue par cooptation. Un lien familial ou amical avec un membre de l'administration constitue quasiment un prérequis.

Dans la mesure où la bureaucratie a été longtemps dominée par les nationalistes, de 1974 à 2005 puis de 2009 à 2013, une carte de membre de l'UBP, du DP ou des réseaux partisans qui vont avec, ont historiquement facilité l'accès aux institutions d'État. Les services de renseignement et le ministère de l'Intérieur effectuent systématiquement des enquêtes préalables sur le profil des candidats voulant accéder aux postes «sensibles $»^{50}$. Sami Dayioğlü, secrétaire pour les affaires internationales du parti de la Démocratie commune (Toplumcu Demokrasi Partisi, TDP), considère ainsi que «le CTP n'a rien fait pour remettre en cause le clientélisme ambiant dans l'administration. Il a utilisé les mêmes procédés que l'UBP et le DP pour donner des postes à ses collaborateurs. ${ }^{51} \gg$ Murat Kanatli est encore plus critique :

Le CTP a menti aux gens. Ils avaient dit qu'ils réformeraient le clientélisme dans les administrations. Mais personne n'a vu le changement. Une fois au pouvoir, ils ont cherché à augmenter leur influence en cooptant leurs amis. Ils disent que la bureaucratie est aujourd'hui plus «pluraliste» qu'avant. C'est peut-être vrai. Mais ce qui n'a pas changé, c'est que ce sont toujours les soutiens familiaux et l'appui d'un parti qui est au pouvoir, qui déterminent l'accès à la fonction publique. Les gens compétents issus de la société civile qui n'ont pas le soutien d'un mentor, les jeunes des classes populaires et les partisans de la réunification ont encore énormément de mal à accéder à la fonction publique sur la base de

50. Ibid.

51.53 ans, Lefkoşa, 8/2/2012. 
leurs mérites. C'est pour cela qu'une bonne partie de nos forces vives préfèrent émigrer ${ }^{52}$.

Pour mieux comprendre ces tendances, le témoignage de Mustafa Tolga, obtenu grâce au rôle d'interprète de sa petite-fille Bürçü, est ici particulièrement précieux. Ancien membre du ministère de l'Intérieur et des services de renseignement, il est désormais secrétaire général de l'association des retraités du secteur public chypriote turc (Kibris Türk Emekliler Cemiyeti). Son parcours fait donc de lui un observateur averti du fonctionnement des institutions de la RTCN, à la fois proche du pouvoir nationaliste, mais aujourd'hui plutôt en retrait de la scène politico-administrative. Il concède que, même si « ce n'est pas toujours le cas», il est vrai que «pour travailler dans le secteur public, mieux vaut soutenir le gouvernement ${ }^{53}$ ! » Pour lui, « en théorie, les recrutements sont transparents », même s'il déclare par la suite: «je ne vous cache pas que ce n'est pas toujours le cas ». Considérant qu' «il y a plusieurs manières d'entrer dans la fonction publique », il reconnaît que «si un membre de votre famille est assez haut placé, cela aide forcément ! » Pour autant, au-delà de ses premières prises de position assez nuancées, le fond de sa pensée émerge peu à peu au fil de notre entretien. Il finit ainsi par me confier qu'il juge négativement le pluralisme politique au sein de l'administration, qui relève selon lui de la « politisation » :

Si la fonction publique est trop politisée, c'est un problème. Il faut exclure ceux qui sont tout le temps en train de s'opposer à tout. Ce n'est pas bon pour le fonctionnement des institutions. Il faut des gens qui pensent de la même manière, dans le sens de la défense de l'intérêt général ${ }^{54}$.

Loin de critiquer la politisation de la bureaucratie entendue comme une trop faible différenciation entre le pouvoir politique et la neutralité théorique de l'administration, il justifie la nécessité d'une certaine homogénéité idéologique dans la sélection du personnel afin d'assurer le bon fonctionnement des institutions. De là à dire qu'il n'y a pas de place pour les critiques et les opposants à la ligne officielle du statu quo au sein de l'administration, il n'y a qu'un pas.

L'accès à la fonction publique crée en ce sens une logique d'appartenance à un corps qui permet de légitimer socialement le projet d'étatisation. Dans la

52. $14 / 2 / 2012$.

53. $13 / 2 / 2012$.

54. Ibid. 
mesure où la RTCN demeure un État non reconnu, les pratiques et le travail des agents du service public ont une vocation performative: elles contribuent en elles-mêmes à faire exister cet État et son administration. Un tel contexte favorise donc directement le ralliement des membres des corps d'État au projet de défense des intérêts des Chypriotes turcs et à l'objectif de construction nationale. Cette situation spécifique liée à la persistance du conflit renforce ainsi la tendance à l'homogénéisation des idéaux politiques des membres de l'administration et des différents corps de l'État, et ce d'autant plus que les nationalistes dominent largement les directions administratives.

La politisation des institutions conduit à la coexistence entre des procédures instituées de promotion et des pratiques de cooptation sur des bases partisanes. Les salaires des fonctionnaires sont régulés par la loi et dépendent de l'avancement (barem) et d'évaluations par la hiérarchie (sicil) qui, même si elles sont sujettes à des pratiques de corruption, montrent l'existence d'un système de régulation ${ }^{55}$. Mais cette procédure encadrée par la loi, qui s'inspire des pratiques des États modernes, coexiste avec des promotions basées sur l'influence sociale et les alliances politiques. Ce dualisme entre l'existence de procédures méritocratiques formelles et des dynamiques de cooptations politiques explique, malgré leur attachement aux postes bureaucratiques, le cynisme, l'ironie et le détachement des Chypriotes turcs à l'égard de leur administration et de leur État. Les sympathisants de l'opposition se plaignent ainsi de ne pas se voir offrir les mêmes opportunités que ceux qui soutiennent les partis nationalistes. Ils adoptent des attitudes de réserve et évitent de parler politique en public. Ceux qui se détachent de l'idéologie nationaliste officielle privilégient ainsi des attitudes d'évitement du politique, marquées par une volonté de ne pas donner son opinion ni se prononcer avec ses collègues ou sa hiérarchie en formulant des jugements de valeur. Ipek, une amie de Bürçü qui travaille aussi dans les services du ministère de l'Intérieur, me raconta une anecdote très éclairante à ce propos :

Il y a deux ans, pendant mes congés, Kadri, l'un de mes collègues, débarqua à l'improviste chez moi. Il prétexta qu'il voulait juste prendre des nouvelles. On discuta de banalités puis il commença à parler de politique et à me demander mon avis. J'étais un peu gênée. Je ne voulais pas parler de ça avec lui de peur que cela m'attire des problèmes. Mais devant son insistance, je me suis dit que si je ne disais rien, cela pourrait se retourner contre moi. J'avais bien compris qu'il était nationaliste par son discours. Je lui ai dit ce qu'il 
voulait entendre et on s'est dit à bientôt en bons termes. Quelques mois plus tard, on m'a proposé une promotion. J'ai compris ensuite qu'il avait sûrement été envoyé par mes supérieurs pour me tester ${ }^{56}$.

Sans le soutien personnel d'un agent, il est de même difficile d'obtenir un document ou une autorisation d'une administration d'État. Sans « connexion », le rapport à l'administration est beaucoup plus difficile, que ce soit pour payer une facture, obtenir un rendez-vous à l'hôpital d'État de Lefkoşa ou renouveler ses papiers d'identité. Loin de constituer un système fermé et limité à une élite, le système du torpil constitue donc un moyen avec lequel les Chypriotes turcs subvertissent leur propre administration en obtenant des faveurs pour eux ou pour leurs proches. «C'est notre seul pouvoir », me dira Bürçü. Mais le système du torpil contribue toutefois à polariser la société entre ceux qui bénéficient d'un capital social et relationnel et peuvent s'appuyer sur celui-ci dans leur vie quotidienne pour obtenir des avantages en nature, des passe-droits et des biens matériels, et ceux qui en sont dépourvus. Les réseaux liés à la distribution des biens et services se construisent et se pérennisent comme une extension de la cellule familiale sur le modèle de la famille élargie. Étant donné que l'acquisition de ce type de capital social et l'accès à la bureaucratie d'État sont intimement liés aux réseaux partisans, et plus particulièrement, aux organisations nationalistes, nombreux sont ceux qui s'affilient à un parti non par conviction, mais dans le but instrumental d'accéder à l'administration. Certains dirigeants créent même des petits partis éphémères suivant des stratégies de «factionnalisme coopératif», dont le but est uniquement de créer un rapport de force pour négocier l'obtention ultérieure d'un poste institutionnel en échange de leur ralliement au parti au pouvoir ${ }^{57}$.

\section{Soumission à l'autorité et résignation sociale}

En me raccompagnant à la suite de mon entretien avec M. Tolga, Bürçü me confia :

Faire partie de l'administration est un privilège. Même avec un petit salaire, avec l'aide des parents, on s'en sort bien. Moi-même, je dois mon poste à mon grand-père. Donc vous savez, je ne dis rien avec ses anciens collègues. J'essaie de ne pas me faire remarquer. J'ai un poste enviable, mais je fais profil bas. C'est un peu « prends ton

56. 31 ans, fonctionnaire du ministère de l'Intérieur, rue Sarayonü, Lefkoşa, 13/2/2012. 57. BOuCEK, 2009, p. 473. 
salaire et tais-toi ». Cela fait trois ans que je travaille au ministère. Je me suis habitué à obéir sans discuter ${ }^{58}$.

Elle avait voulu réagir à des prises de position parfois très tranchées de son grand-père. À travers son attitude de soumission envers sa hiérarchie, elle traduisait «l'ambivalence du don » et plus particulièrement, la facette de «la dette personnelle qui enchaîne » pour reprendre l'expression de l'ethnographe Florence Weber, qui induit dans son cas un certain effacement de soi ${ }^{59}$. Les psychologues sociaux ont bien montré que la soumission à l'autorité se fonde sur une acceptation de la hiérarchie, l'intériorisation par l'acteur de la faiblesse de sa position de pouvoir, et se perpétue grâce à la sécurité et la stabilité psychique procurées par le respect des ordres et la mise en ouvre de tâches d'exécution qui n'induisent que peu de responsabilités. L'accès à l'administration à travers le système du torpil, grâce aux relations familiales et amicales (eş-dost iliş̧kisi), engendre une obligation de rendre qui, devant l'impossibilité d'effectuer un «contre-don» instrumental équivalent, génère une dépendance qui implique loyauté et intériorisation d'attitudes de déférence. La soumission à l'autorité qui prévaut dans la structure du pouvoir et les pratiques sociales des administrations a aussi pour effet d'évincer de la fonction publique les jeunes diplômés compétents qui feraient preuve d'attitudes jugées trop anticonformistes. L'administration civile demeure encore aujourd'hui contrôlée par la génération des anciens combattants de la TMT et par leurs descendants, dont la loyauté à l'égard de l'État leur permet de contrôler les positions dominantes, parfois sans forcément être dotés de formations spécifiques. Les jeunes diplômés qui veulent progresser au mérite sans entrer dans le jeu du torpil, des codes informels, des réseaux et de l'allégeance, se voient donc dans l'incapacité d'accéder à un poste dans un secteur public fondé sur la valorisation d'attitudes de conformisme, de suivisme et de soumission à l'autorité.

L'administration nord-chypriote est une structure de pouvoir, à savoir une institution dans laquelle les fonctionnaires, à chaque échelon, possèdent une parcelle d'autorité exercée théoriquement dans le cadre de la fonction publique. Globalement, les agents de base ne détournent pas ce pouvoir à des fins privées pour bénéficier d'un enrichissement illicite. La corruption est surtout l'apanage des élites administratives et politiques. Pour le haut-fonctionnaire, le « jeu des relations » peut aller jusqu'à obtenir des faveurs en violation de la réglementation

58. Bürçü, 26 ans, fonctionnaire du ministère de l'Intérieur, rue Sarayonü, Lefkoşa, $13 / 2 / 2012$.

59. WeBER, 2007, p. 58. 
en vigueur, ce qui signifie que la puissance du réseau peut être au-dessus de la réglementation. Elle s'exprime à travers les augmentations de salaires des dirigeants, les collusions familiales et partisanes avec des acteurs du secteur privé lors de l'attribution des marchés publics, et l'existence de certains cas de rétrocommissions dénoncées par la presse d'opposition ${ }^{60}$. Le fonctionnaire de base bénéficie quant à lui d'avantages en nature et peut abuser de la sécurité que lui procure sa fonction pour améliorer sa qualité de vie, ce qui contribue à accroître la distinction de sa position par rapport aux autres membres de la société. Il peut arriver en retard, prendre des jours de congé sans prévenir, quitter son poste quelques minutes, voire plusieurs heures, à l'avance, prendre son après-midi en invoquant différents prétextes et se rendre à la plage durant les mois d'été, ou encore utiliser son véhicule de fonction pour son usage personnel ${ }^{61}$. Lors de mon entretien avec Selim, assistant au ministère des Affaires étrangères, il évoqua par exemple la «disparition » sans raison de son chef pendant une semaine l'année précédente, puis sa tentative de se justifier au motif d'un voyage d'affaires, ou encore sa tendance à s'absenter pendant deux ou trois heures lors de la pause du midi ${ }^{62}$.

Ces pratiques sociales engendrent un certain conformisme, une indifférence à l'égard de leur travail, et des sentiments de dévalorisation et d'apathie (isteksizlik) chez de nombreux agents, particulièrement dans l'administration ${ }^{63}$. Les fonctionnaires utilisent des phrases ironiques comme: «laisse passer le mois, laisse l'argent arriver » ou encore «la fin du mois est derrière la porte », pour dire qu' ils obtiendront bientôt leur salaire sans avoir trop travaillé6 ${ }^{64}$. Lorsque j'ai rencontré à nouveau Bürçü quelques jours plus tard pour une conversation plus longue autour d'un thé sur la place Inönü de Lefkoşa, elle reconnut que certains de ses collègues abusent du système et se sont habitués à recevoir leur salaire sans trop d'efforts :

Certains arrivent systématiquement en retard. D'autres prennent des jours de congé sans prévenir. L'été c'est très fréquent. Ils se rendent à la plage et disent ensuite qu'ils étaient malades. Souvent mon chef fait la sieste dans son bureau en début d'après-midi. Il se rend souvent à des «rendez-vous» en ville,

60. Entretien avec Şener Levent, Cyprus Mail, 2019.

61. Entretiens avec Ipek et Bürçü, 13/2/2012.

62. Lefkoşa, 20/3/2014.

63. Navaro-Yashin, 2012, p. 85.

64. Ibid.p. 83. 
mais en fait, on ne sait pas où il est! Personne ne dit rien. Tout le monde profite du système d'une manière ou d'une autre ${ }^{65}$.

Selon elle, certains sont même embauchés sans avoir de réelles fonctions et errent dans les bureaux à la recherche de petites tâches que leur donnent les chefs de service. Sur ce point, Yael Navaro-Yashin évoque la figure du «messager » (odacı), dont le rôle « est de transporter les papiers d'un bureau ou d'un service à un autre au sein de l'administration d'État ${ }^{66} \gg$.

Cette figure de l'odacı est ici assez similaire à celle du chaouch dans l'administration tunisienne, dont les fonctions sont directement issues de l'administration ottomane. Les fonctionnaires de base contribuent non seulement au bon fonctionnement du travail administratif en classant des documents, en apportant des dossiers ou en accueillant les visiteurs, mais comme le note Béatrice Hibou, «du fait des pratiques sociales et de la nature des relations de pouvoir, ils participent simultanément à la surveillance de leurs collègues et à l'instauration d'une "discipline de service" ${ }^{67} \gg$. Au quotidien, cette pratique routinière de la surveillance va de pair avec une reconnaissance par la hiérarchie et des opportunités de promotion pour les acteurs «loyaux» n'hésitant pas à dénoncer les conduites déviantes de leurs collègues. Cela favorise toutefois « une logique de l'inaction », une certaine lassitude, des attitudes d'évitement du conflit et de dépolitisation des agents qui cherchent seulement à conserver leur poste ${ }^{68}$. En résumé, la prégnance du «dilettantisme », pour reprendre l'expression d'Ahmed Rouadjia à propos de la bureaucratie algérienne, accentue non seulement l'inefficacité des administrations, mais médiatise aussi et surtout un élément de distinction des privilèges sociaux des fonctionnaires, dont la tendance à abuser du système est inversement proportionnelle à leur position hiérarchique ${ }^{69}$.

Pour Kerem Dandelen, journaliste du quotidien d'opposition Yeni Düzen à Famagouste, l'administration serait en fait à l'image d'un État fondé sur la base de l'aide turque et du pillage (ganimet) des biens des Chypriotes grecs.

Après 1974, Rauf Denktaş se rendait dans les villages pour inciter les citoyens à s'approprier sans complexe les biens des

\author{
65. 13/2/2012. \\ 66. Navaro-Yashin, 2006, p. 283. \\ 67. Нiвоu, 2006, p. 133. \\ 68. Ibid., p. 136. \\ 69. Rouadjia, 1994, p. 277-282.
}


Chypriotes grecs. L'État qu'il a construit suit la même logique. Il est au service de ses agents et non des citoyens. Ceux qui accèdent à l'administration grâce à leurs réseaux se comportent ensuite comme des prédateurs. Ils essaient d'obtenir le plus d'avantages matériels ou en nature. Ils utilisent leurs « connexions » pour faire avancer tel ou tel dossier. Ils font embaucher leurs enfants. Ce sont les agents du statu quo ${ }^{70}$.

Selon elle, les avantages en nature et la culture du dilettantisme qui prévaudraient au sein des administrations constitueraient «une manière de compenser les dysfonctionnements de l'État», des comportements acceptés socialement car vécus comme «une compensation pour le maintien de la domination de l'armée turque et l'absence de perspectives ${ }^{71} \gg$. Les Chypriotes turcs sont en effet pleinement conscients de leur dépendance à l'égard de la Turquie et de l'armée turque qui contrôle leur territoire. Les fonctionnaires de police et de la justice font quotidiennement l'expérience des limites de la «souveraineté » autoproclamée de leur entité. Lors d'une matinée d'observation au service des passeports du ministère de l'Immigration, après avoir sympathisé avec Volkan, un agent administratif, il me confia ainsi lors de sa pause-café après l'avoir questionné sur son travail :

Ici, on s'occupe de délivrer les cartes de séjour, les papiers d'identité et les passeports. Pour les renouvellements, c'est une procédure administrative simple. Le côté intéressant est lié à la délivrance ou non de la citoyenneté pour les immigrés turcs qui le demandent et qui respectent les conditions de résidence. Nous avons des critères très clairs et bien définis qui nous amènent à rejeter des demandes. Mais avant les élections, nos supérieurs nous incitent parfois à être plus flexibles pour « régulariser» plus de Turcs comme ils disent. J'ai souvent l'impression de travailler en vain. On nous donne des critères, puis on nous dit de ne pas les appliquer $^{72}$.

70.42 ans, Karpas Caddesi, Gazimağusa, 1/4/2014.

71. $1 / 4 / 2014$.

72. Lefkoşa, 3/3/2014. 
Le même type de sensation fut mis en avant par Ismaïl, un policier à la retraite, lors d'une longue discussion à la terrasse d'un café du centre-ville historique de Famagouste:

Lorsque j'étais policier, mon travail était de faire des patrouilles, d'enquêter suite aux délits ou encore d'interpeller les étrangers en situation irrégulière. Mais très souvent, nous n'avions pas le dernier mot. C'est un peu décourageant. C'est l'armée turque qui décide. Si nous interpellons des immigrés illégaux, mais que l'armée ne contrôle pas les frontières et les allées et venues au port de Famagouste en provenance de Turquie, notre travail ne sert à rien. La décision de poursuivre en justice un individu interpellé est prise par l'armée et non par la police de la RTCN. La police ici n'est pas autonome. Ses ordres viennent de l'armée turque ${ }^{73}$.

Ces témoignages illustrent bien l'ambivalence des rapports des agents de l'État avec leur administration dans le contexte spécifique d'un État de facto non reconnu. Leur déférence formelle à l'égard de la Turquie se double d'un certain ressentiment à l'égard de sa domination. Le respect des procédures formelles de l'administration dont ils font eux-mêmes partie et dont ils sont dépendants, va de pair avec une dépréciation du caractère factice de leur propre État, ressenti notamment par l'impression de «travailler en vain », ce qui génère malaise et résignation.

\section{Conclusion}

Cet article a posé la problématique des rapports qu'entretiennent les Chypriotes turcs et plus particulièrement les agents de l'État avec l'administration de leur territoire non reconnu. Il montre que l'exclusion de la RTCN des relations internationales formelles, la persistance du conflit et l'isolement du territoire ont favorisé chez eux l'institutionnalisation d'une situation de dépendance à l'égard de leur administration, elle-même dépendante du soutien politico-militaire de la Turquie. Ce contexte alliant militarisation de l'espace, impasse politique et précarité sociale des citoyens, explique ainsi la déférence et la loyauté des acteurs de l'État à l'égard de leur administration, même s'ils sont pleinement conscients des limites et du caractère quelque peu factice de sa souveraineté. L'ethnographie de l'État proposée ici a donc d'abord permis de refléter les modalités de légitimation sociale et de persistance d'une administration pourtant ignorée par la politique

73. Rue Liman Yolu, Gazimağusa, 2/4/2014. 
et le droit international, en illustrant sa fonction cruciale d'employeur dans un contexte de précarité économique et d'isolement diplomatique. Loin de délégitimer l'État aux yeux de ses citoyens, l'isolement international et l'embargo économique subis par la RTCN ont renforcé leur dépendance à l'égard d'une administration dont ils sont pourtant les premiers à critiquer les dysfonctionnements. La souveraineté autoproclamée, mais imparfaite, du territoire dans lequel ils vivent, explique que les Chypriotes turcs ont dû s'accommoder d'une dépendance à l'égard de la Turquie, d'une reconnaissance presque forcée à son égard et d'une bureaucratie partiellement autonome, dans le cadre de rapports ambigus de domination. Cette étude a ainsi permis de montrer que la légitimation sociale d'une autorité pourtant jugée «illégitime » s'explique comme une stratégie de survie et une réponse sociale imparfaite à une situation réelle d'isolement politique pour des gens qui, depuis quarante ans, ont dû apprendre à vivre sans reconnaissance internationale.

L'ethnographie des représentations que se font les Chypriotes turcs de leur propre entité politique illustre de plus, dans la veine de travaux récents d'anthropologie politique, que l'État ne se résume pas simplement à un système institutionnel, mais doit aussi être considéré comme «affectif» dans la mesure où ses actions et sa bureaucratie génèrent des émotions spécifiques chez ses agents et parmi ses citoyens. À Chypre du Nord, l'«État» produit schizophrénie et malaise puisqu'il gouverne, émet des normes et vote des lois, mais demeure aussi «fictif» tant sa souveraineté demeure dépendante du monopole de la violence imposé par l'armée d'un autre État. La conscience par les fonctionnaires chypriotes turcs des limites de leurs actions en l'absence d'une autorité politique pleinement souveraine, génère insatisfaction, démotivation et des attitudes de résignation. Le contexte d'une double relation de dépendance, des agents publics vis-à-vis de leur administration, elle-même dépendante à l'égard de la Turquie, favorise l'acceptation contrainte de relations clientélistes avec la bureaucratie et une politisation des institutions. Mais ce rapport des Chypriotes turcs avec leur État non reconnu crée aussi malaise, insatisfaction chronique et une ambivalence de leurs sentiments, puisqu'ils se trouvent imbriqués et confinés dans une situation de dépendance qui contraint leurs attitudes et leur vie quotidienne. Dans l'impossibilité «de rendre» le soutien instrumental qu'apportent l'appui de la Turquie et leur propre administration, ils en sont donc devenus les obligés à travers une reconnaissance symbolique, une allégeance tacite au nationalisme turc et l'intériorisation d'attitudes de soumission à l'autorité. L'ethnographie d'un État non reconnu permet enfin d'illustrer le caractère performatif et fragile à la fois de l'État lui-même: en 
50 Politique et société à Chypre aujourd'hui

dépit de ses ambivalences, la bureaucratisation de la RTCN contribue à « faire exister » cette entité malgré les limites objectives de sa souveraineté.

\section{Bibliographie}

\section{Monographies}

Attalides Michael, 1979, Cyprus: Nationalism and International Politics, Q Press, Edinburgh, 226 p.

BoAs Franz, 1897, The Social Organization and the Secret Societies of the Kwakiutl Indians, Based on Personal Observations and on Notes Made by Mr. George Hunt, Harvard University Press, Washington, 738 p.

Bourdieu Pierre, 1991, «L'économie des biens symboliques » in Raisons pratiques, Le Seuil, Paris, p. 27-51.

Bryant Rebecca, 2004, Imagining the Modern. The Cultures of Nationalism in Cyprus, I. B. Tauris, London, $256 \mathrm{p}$.

Caillé Alain \& Godbout Jacques, 1992, L’Esprit du don, La Découverte, Paris, $364 \mathrm{p}$.

Copeaux Étienne \& Mauss-Copeaux Claire, 2005, Taksim! Chypre divisée (1964-2005), Éditions Aedelsa, Lyon, 235 p.

Dubors Vincent, 1999, La Vie au guichet. Relation administrative et traitement de la misère, Economica, Paris, 224 p.

Godelier Maurice, 1996, L'Énigme du don, Grasset, Paris, 315 p.

Hibou Béatrice, 2006, La Force de l'obéissance. Économie politique de la répression en Tunisie, La Découverte, Paris, 372 p.

Loizos Peter, 2008, Iron in the Soul. Displacement, Livelibood and Health in Cyprus, Berghahn Books, London, 224 p.

Malinowski Bronisław, 1989 [1922], Les Argonautes du Pacifique occidental, Gallimard, Paris, 606 p. 
Mauss Marcel, 2007 [1924-1925], Essai sur le don, Gallimard, Paris, 252 p.

MÉDARD Jean-François (dir.), 1992, États d'Afrique: formations, mécanismes, crise, Karthala, Paris, 405 p.

Navaro-Yashin Yael, 2012, The Make Believe Space. Affective Geography in a Postwar Polity, Duke University Press, Durham, 296 p.

Patrick Richard, 1976, Political Geography and the Cyprus Conflict 1963-1971, Department of Geography Publication Series, n 4, Waterloo, 481 p.

Peg G Scott, 1998, International Society and the de facto State, Ashgate, Aldershot, $322 \mathrm{p}$.

Rouadjia Ahmed, 1994, Grandeur et décadence de l'État algérien, Karthala, Paris, $406 \mathrm{p}$.

UĞural Çetin \& Sabit Soyer Ferdi, 1998, Sükran Ekonomisi: Üzerimizdeki Ipotek, Kuzey Kıbrıs Ekonomisi Bütçe Analizleri, Naci Talat Vakfı Yayınları, Nicosia.

Weiner Annette, 1992, Inalienable Possessions. The Paradox of Keeping While Giving, University of California Press, Berkeley \& Los Angeles, 232 p.

\section{Articles et participations à des ouvrages collectifs}

Bertrand Gilles, 2000, « Chypre : diaspora(s) et conflit », Cabiers d'études sur la méditerranée orientale et le monde turco-iranien, $\mathrm{n}^{\circ} 30$, p. 59-81.

Boucek Françoise, 2009, "Rethinking Factionalism: Typologies, Intra-Party Dynamics and Three Faces of Factionalism", Party Politics, ${ }^{\circ}$ 4, vol. 15, pp. 470-485.

Fassin Didier, 2013, "On Resentment and Ressentiment. The politics and ethics of moral Emotions", Current Anthropology, n 3, vol. 54, pp. 249-267.

KiziLyÜrek Niyazi, 2002, "National Memory and Turkish Cypriot Textbooks", in Koulouri Christina (ed.), Clio in the Balkans. The Politics of History 
52 Politique et société à Chypre aujourd'hui

Education, Center for Democracy and Reconciliation in Southeast Europe, Thessaloniki, pp. 432-435.

Lagroye Jacques \& Siméant Johanna, 2003, «Gouvernement des humains et légitimation des institutions $\gg$ in FAVRe Pierre, HaYward Jack \& Schemeil Yves (dir.), Etre gouverné. Études en l'honneur de Jean Leca, Presses de Sciences Po, Paris, p. 54-56.

Navaro-Yashin Yael, 2006, "Affect in the civil service: A study of a modern state System", Postcolonial Studies, no 3, vol. 9, pp. 281-294.

Papadakis Yiannis, 1993, "The Politics of Memory and Forgetting in Cyprus", Journal of Mediterranean Studies, $\mathrm{n}^{\circ}$ 1, vol. 3, pp. 141-162.

Rosbapé Philippe, 1993, «Don archaïque et monnaie sauvage », La Revue $d u$ MAUSS, $\mathrm{n}^{\circ} 1$, p. 15-33.

Stoler Ann Laura, 2004, "Affective States", in Nugent David \& Vincent Joan (eds.), A Companion to Anthropology of Politics, Blackwell Press, London, pp. 4-20.

Weber Florence, 2007, «Vers une ethnographie des prestations sans marché » in Essai sur le don, PUF, Paris, p. 23-40.

Résumé: cet article propose une étude ethnographique des dynamiques d'attachement des Chypriotes turcs à l'égard de leur administration, dans le contexte d'un État de facto, non reconnu, et dépendant de la Turquie depuis la partition de Chypre en 1974. En s'appuyant sur des enquêtes de terrain menées entre 2012 et 2014 et dans une approche d'anthropologie politique, il s'intéresse aux affects générés par la « République turque de Chypre du nord » sur ses agents publics et ses citoyens. Il montre que l'isolement diplomatique et économique renforce leur dépendance et loyauté à l'égard de leur administration, mais engendre aussi malaise et insatisfaction chronique chez des citoyens conscients des limites de la «souveraineté » autoproclamée de leur entité politique.

Mots-clefs: Chypre du Nord, État de facto, anthropologie politique, République de Chypre, 1974: débarquement turc à Chypre, 1983 : proclamation de la «République turque de Chypre du Nord », sociologie politique 
This paper proposes an ethnographic study of Turkish Cypriots' dynamics of attachment towards their administration, in the context of an unrecognized de facto State dependent upon Turkey since Cyprus' partition in 1974. On the basis of several fieldworks conducted between 2012 and 2014 and through an approach of political anthropology, it studies the affects generated by the "Turkish Republic of Northern Cyprus" on its public agents and citizens. It shows that the diplomatic and economic isolation reinforces their dependence and loyalty towards their administration. But it also generates malaise and chronic dissatisfaction on citizens entirely conscious of the limits of the self-proclaimed sovereignty of their political entity.

Keywords: Northern Cyprus, de facto State, political anthropology, Cyprus Republic, 1974: Turkish landing in Cyprus, 1983: proclamation of the «Turkish Republic of Northern Cyprus», political sociology

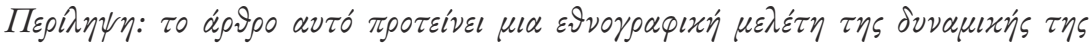

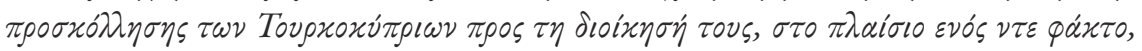

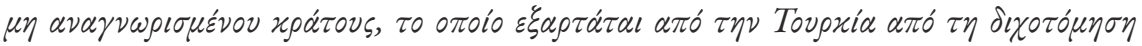

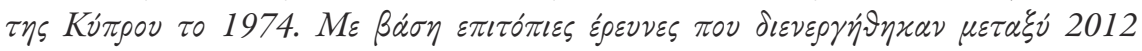

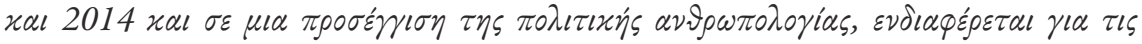

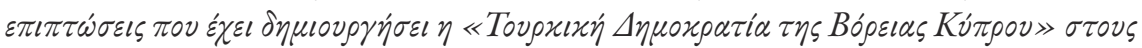

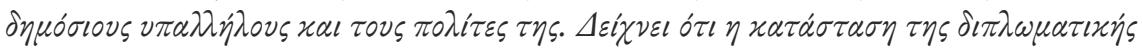

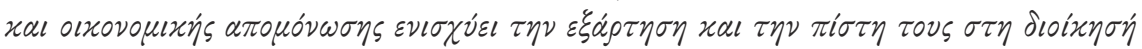

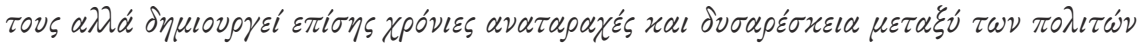

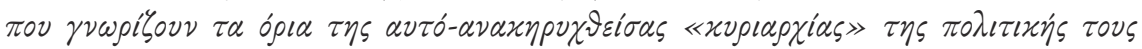

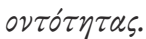

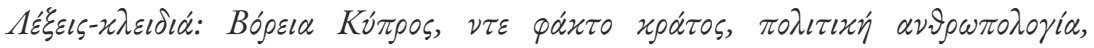

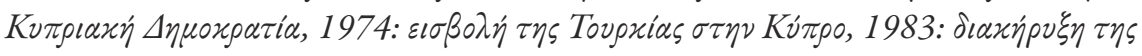

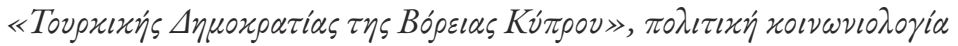

Anahtar Kelimeler: Kuzey Kibris, gerçek devlet, siyasi antropoloji, Kibris Cumburiyeti, 1974: Kibris'ta Attila operasyonu, 1983: «Kuzey Kıbrıs Türk Cumburiyeti» nin İlant, siyaset sosyolojisi

Клучни зборови: Северен Кипар, де факто држава, политичка антропологија, Република Кипар, 1974 година: операџија на Атила во Кипар, 1983: прогласувате на «Турската Република Северен Кипар», политичка сочиологија 